Mini-Review

DOI: $10.36959 / 961 / 627$

\title{
Chemistry and Quantum
}

Ogilvie $\mathrm{JF}^{1,2,3^{*}}$

${ }^{1}$ Department of Mathematics, Centre for Experimental and Constructive Mathematics, Simon Fraser University, Canada ${ }^{2}$ Escuela de Quimica, Universidad de Costa Rica, Ciudad Universitaria Rodrigo Facio, San Pedro de Montes de Oca, San Jose, Costa Rica

${ }^{3}$ Institute of Quantum Physics, Irkutsk National Research Technical University, Russian Federation, Russia

\begin{abstract}
We consider the quantum aspects of chemical and physical observations and operations, including quantum physics, quantum mechanics, quantum chemistry and the quantum laws of nature.
\end{abstract}

\section{Introduction}

The technical term quantum implies discrete - the discreteness of a physical entity or an observable property. This term might appear in four legitimate scientific contexts - quantum physics, quantum mechanics, quantum chemistry and quantum laws. As an extension of a previous report [1], we consider briefly each in turn.

Quantum physics signifies the observation and analysis of discrete phenomena. A perhaps most obvious instance of a discrete nature is a narrow spectral line. The line at wave length $21 \mathrm{~cm}$, subject to a red shift depending on the relative speed of emitting $\mathrm{H}$ atom and observer, originating in interstellar clouds of atomic hydrogen lies toward one extreme of the natural electromagnetic spectrum, and x-rays or $\gamma$-rays toward the other extreme. Such discrete phenomena are not limited to visual observations. An analysis of those spectral lines provides information about the properties of their carriers. In 1924 Heisenberg wrote that the observable properties of (free) atoms and molecules are the frequencies and intensities of their spectral lines; their analysis yields information about primarily the energies of discrete states, and secondarily about the structures and other properties of the carriers of those lines. A Geiger counter placed near a weakly radioactive material can produce occasional clicking sounds through its speaker, each of which indicates the detection of a decomposition of a single unstable atomic nucleus. An analysis of the rate of audible clicking sounds from a Geiger counter might yield an estimate of the halflife of the emitter, which can lead to its identification through reference to standard tables of such data.

Quantum mechanics is now recognised to comprise a collection of methods of calculations, or algorithms, that are applicable to systems on an atomic scale. At least thirteen such methods are known to have been developed [1], although only a few are applied in general practice. The significance of these multiple methods is that each might possess its particular artifacts, such as orbitals in wave mechanics, which are invaluable within a calculation pertaining to a particular method but which fail to constitute observable properties. For chemists wave mechanics is practically the only method of general application for atomic and molecular properties, but that method fails to be directly applicable in the case of a calculation of some properties related to nuclear-magneticresonance spectra because nuclear spins lack an associated positional coordinate; in such a case diagonalisation of a matrix [2], in the spirit of matrix mechanics, yields the desired pattern of spectral lines based on the chemical shifts and coupling parameters.

Quantum chemistry is generally associated with a practice of calculations with computer programs designed to yield results for observable molecular properties, such as the geometric structure at an atomic level, electric-dipolar moments and spectral frequencies and intensities of lines associated with transitions between molecular states. These programs, which have become highly developed, require little or no knowledge or understanding of the underlying principles of wave mechanics on which they are roughly based. Especially during the earlier years of such calculations,

*Corresponding author: Ogilvie JF, Department of Mathematics, Centre for Experimental and Constructive Mathematics, Simon Fraser University, Canada; Escuela de Quimica, Universidad de Costa Rica, Ciudad Universitaria Rodrigo Facio, San Pedro de Montes de Oca, San Jose, Costa Rica; Institute of Quantum Physics, Irkutsk National Research Technical University, Russian Federation, Russia

Accepted: November 25, 2021

Published online: November 27, 2021

Citation: Ogilvie JF (2021) Chemistry and Quantum. Ann Atoms Molecules 3(1):14-16 
the authors of articles presenting their results described them as being $a b$ initio or from first principles, but such a portrayal is laughable - for various reasons. First of all, even Schroedinger himself recognised that his equation was not correct relativistically, which means that there is in principle an inherent deficiency in any result from a calculation on that basis. Secondly, in those programs only the electronic motions and the electronic density are directly treated; a rigorous treatment would include the corresponding nuclei. Quantum mechanics is incompatible with molecular structure in that, as a result of a rigorous calculation treating electrons and atomic nuclei in an equitable manner, at the end of a calculation no classical structure is discernible [3], apart from trivial cases of diatomic molecules for which the nuclei are invariably distinguishable from the associated electrons. Thirdly, those programs incorporate canned basis sets, which have been developed to reproduce selected properties in a calibrated manner; a basis set designed to reproduce one property, such as molecular structure, might not be optimum for the reproduction of another property, such as a chemical shift. Under these conditions these programs must be considered semi-empirical. With the extraordinary power of our contemporary computers that operate these programs, a practicable basis set might even approach the Hartree-Fock limit, and provisions to take account of electronic correlation are equally practicable, but in principle the calculations must still be considered approximate, despite the utility of the results for comparison with, or interpretation of, experimental data. Some quantum-chemical programs are even based on the Dirac equation to take account of relativistic effects, but, in all cases of a significant number of atoms, a trial molecular structure must be input before a calculation can proceed to optimise the various parameters of interest. Furthermore, all such calculations inevitably take into account the interactions between constituent particles of a system of interest only pair-wise, so between two particles at a time, whereas one particle naturally interacts simultaneously with all other particles in the system; the effects of this approximation in a calculation are seldom considered.

The quantum laws [4] of nature are best regarded as the laws of discreteness. Five properties - mass, electric charge, energy, linear and angular momenta - are found on an atomic scale to assume discrete values. All these properties on a macroscopic scale are subject to laws of conservation. For instance, the conservations of mass and of net electric charge serve as simple criteria to balance a chemical equation, even though according to a physical point of view mass and energy should formally be treated together. In the energy of the system in Darwin's solution of the hydrogen atom according to Dirac's relativistic wave mechanics [5] the first term is precisely $m_{e} c^{2}$, with $m_{e}$ as electronic rest mass and $c$ as speed of light in vacuum; this term hence implies the intrinsic mass energy of an electron. The linear momentum of a particle assumes discrete values only if that particle is confined to a finite volume, whereas angular momentum and energy are crucial properties of which discrete values define particular discrete states of an atom or molecule and the frequencies and intensities of narrow spectral lines associated with transitions between those states. The application of these quantum laws involving energy and angular momentum to features of molecular spectra is discussed at some length elsewhere [1].

One might inquire about the origins of ideas about the discreteness of the five properties specified above. In London in 1815, an English chemist and physician named William Prout published an hypothesis, based on inaccurate measurements of molar masses of the then known chemical elements, that the hydrogen atom was the only truly fundamental object and that atoms of other chemical elements comprised aggregates of hydrogen atoms of varied number. In 1920 Ernest Lord Rutherford named the nucleus of a hydrogen atom a proton. In Cambridge also in 1920, following his production of the first mass spectrograph in 1919, Francis Aston formulated the whole-number rule whereby the masses of atomic isotopes are practically integer multiples of the mass of the hydrogen atom. In Cambridge in 1932, Sir James Chadwick's discovery of the neutron achieved a simple interpretation of an atomic nucleus of integer mass number $A$ as comprising $Z$ protons and $A-Z$ neutrons, supplanting Prout's hypothesis, but Prout's idea might be considered the basis of the discreteness of mass. J.J. Thomson is credited with the identification of an electron (previously named by Stoney), the first subatomic particle to be discovered; in 1897 in Cambridge he showed that cathode rays were composed of previously unknown negatively charged particles, which he calculated must have sizes much smaller than atoms and a large ratio of charge to mass. The discrete energies of states of atoms and molecules were deduced from application by Walther Ritz of the Rayleigh-Ritz combination principle in conjunction with the attribution of transitions between those states by John Nicholson in Cambridge in 1911.

The discrete radiant energy of a photon, a term originating with G. N. Lewis in 1926, was an outcome of Planck's flawed derivation of an equation to describe the distribution with wave length or frequency of thermal electromagnetic radiation in thermodynamic equilibrium with its environment. Planck's successors subsequently improved the derivation, but it still suffers from the undeniable fact that the distribution of energy emitted by a black body is fundamentally continuous, hence requires no discrete nature of explanation [6]. Einstein's interpretation of the photoelectric effect in 1905 was the first substantive recognition of the discreteness of radiation. In 1917 Einstein obtained [7] Planck's equation for radiation from a black body on considering three processes: $A+\phi \rightarrow A^{*}, A^{*} \rightarrow A+\phi$ and $A^{*}+\phi \rightarrow A+2 \phi$, in which $A$ represents an atom or molecule in its state of lower energy, $A^{*}$ represents that same atom or molecule in an excited state and $\phi$ represents a photon that provides the difference of energy between the energies of the two states. These three processes hence define absorption, spontaneous emission and stimulated emission, respectively. To reproduce Planck's formula Einstein had to include the third process; in so doing he forecast the maser and laser that operate through stimulated emission. Planck's formula, or its reproduction by Einstein, might be considered an approximation, because it fails to take account of a process involving two photons simultaneously incident on an atom or molecule leading 
to absorption, as $A+2 \phi \rightarrow A^{*}$; as such a process requires incident light of great intensity, for practical purposes in a laboratory in relation to radiation from a hot object that process might be neglected, but within the photosphere or core of the sun the severe conditions might be conducive to phenomena not yet articulated. The angular momentum of a photon was described by Poynting in 1909 [8]; the quantised linear momentum of a confined particle originated with Duane in 1923 [9], based on Einstein's preceding recognition of the linear momentum of a photon [7].

The idea of quantum has become an essential and intrinsic component of understanding chemical and physical phenomena, in its contexts of quantum physics, quantum mechanics, quantum chemistry and the quantum laws. For chemical applications, quantum physics in a form of analysis of spectra as a basis of deriving information about molecular structure and other properties is most valuable, whereas the quantum laws can serve to explain the presence of features in such spectra. Quantum-chemical calculations can greatly aid an analysis of such spectra, but quantum mechanics is rarely directly useful.

\section{References}

1. Ogilvie JF (2022) Chemistry and quantum mechanics. Journal of the Chinese Chemical Society 69: 1-7.

2. Laidler KJ, Meiser JH, Sanctuary BC (2003) Physical Chemistry. $\left(4^{\text {th }}\right.$ edn), Houghton-Mifflin, Boston MA USA, 749.

3. Woolley RG (1976) Quantum theory and molecular structure. Advances in Physics 25: 27-52.

4. Ogilvie JF (1990) The nature of the chemical bond-1990: There are no such things as orbitals. J Chem Educ 67: 280-289.

5. Ogilvie JF (2021) Quantum Mechanics for Chemistry.

6. Boyer TH (1969) Derivation of the black-body spectrum without quantum assumptions. Physical Review Journals Archive 182: 1374.

7. Einstein A (1917) On the quantum theory of radiation. Physikalische Zeitschrift 18: 121-128.

8. Poynting J (1909) The wave motion of a revolving shaft, and a suggestion as to the angular momentum in a beam of circularly polarised light. Proceedings of the Royal Society London 82: 560567.

9. Duane $W(1923)$ The transfer in quanta of radiation momentum to matter. Proc Natl Acad Sci USA 9: 158-164.

DOI: $10.36959 / 961 / 627$ 